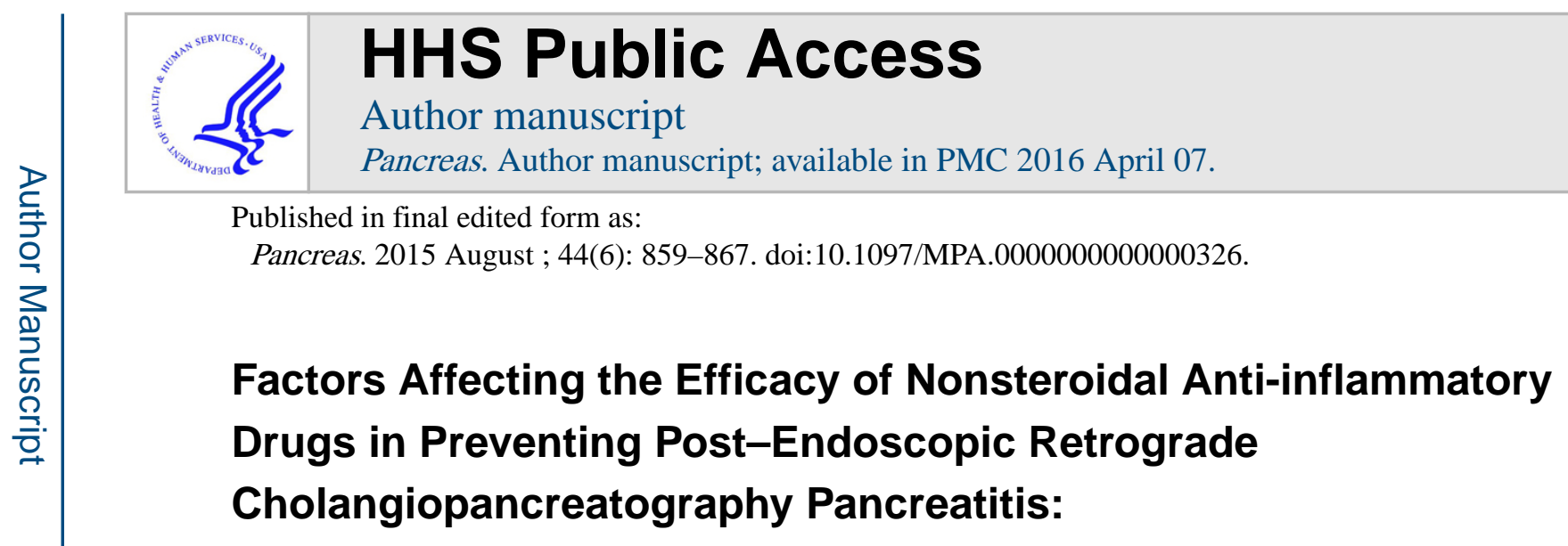

\title{
A Systematic Review and Meta-analysis
}

Tarun Rustagi, MD* and Basile Njei, MD ${ }^{\dagger}$

*Section of Digestive Diseases, Department of Internal Medicine, Yale University School of Medicine, New Haven

†Department of Internal Medicine, University of Connecticut Health Center, Farmington, CT

\begin{abstract}
Objectives-To identify the factors affecting the efficacy of nonsteroidal anti-inflammatory drugs (NSAIDs) in preventing post-endoscopic retrograde cholangiopancreatography (ERCP) pancreatitis (PEP).
\end{abstract}

Methods-We systematically searched databases for relevant studies published from inception to November 2013.

\begin{abstract}
Results-A meta-analysis of 11 randomized trials $(n=2497)$ revealed a significant reduction in PEP in patients who received NSAIDs compared with that in patients who received placebo (relative risk [RR], 0.59; 95\% confidence interval [CI], 0.41-0.85; $P=0.005$ ). In subgroup analysis by treatment type, indomethacin had no significant effect (RR, 0.66; 95\% CI, 0.38-1.15; $P=0.14$ ), whereas other NSAIDs showed significant benefit (RR, $0.51 ; 95 \% \mathrm{CI}, 0.29-0.91 ; P=$ $0.02)$. Only rectal administration significantly reduced the incidence of PEP (RR, $0.43 ; 95 \% \mathrm{CI}$, $0.32-0.58 ; P<0.00001)$. The risk for PEP was the lowest among patients who received NSAIDs before ERCP (RR, $0.48 ; 95 \% \mathrm{CI}, 0.29-0.78 ; P=0.003$ ). NSAIDs did not significantly reduce the risk of PEP in men (RR, 0.61; 95\% CI, 0.34-1.09), patients with sphincter of Oddi dysfunction (RR, $0.98 ; 95 \% \mathrm{CI}, 0.38-2.54$ ), or patients with pancreatic duct injection (RR, 0.64; 95\% CI, $0.35-1.18)$.
\end{abstract}

Conclusions-Rectal administration of NSAIDs (especially diclofenac), before ERCP, seemed to be the most effective strategy for preventing PEP.

\footnotetext{
Reprints: Tarun Rustagi, MD, Section of Digestive Diseases, Department of Internal Medicine, Yale University School of Medicine, 333 Cedar St, 1080 LMP, New Haven, CT 06520-8019 (tarunrustagi06@gmail.com; tarun.rustagi@yale.edu).

The authors declare no conflict of interest.

T.R. contributed to the study concept and design, as well as with the analyses, study preparation and revision. B.N. contributed to the statistical analyses, study preparation, and revision.

Supplemental digital contents are available for this article. Direct URL citations appear in the printed text and are provided in the HTML and PDF versions of this article on the journal's Web site (www.pancreasjournal.com).
} 


\section{Keywords}

pancreatic duct injection; sphincter of Oddi dysfunction; indomethacin; diclofenac; sphincterotomy; rectal

Post-endoscopic retrograde cholangiopancreatography (ERCP) pancreatitis (PEP) is one of the most common and feared complications of ERCP, resulting in considerable morbidity and, rarely, in death. ${ }^{1,2}$ Post-ERCP pancreatitis prevention has remained the focus of clinical and research interventions. Although numerous trials studying a variety of pharmacological agents (eg, octreotide, corticosteroids, allopurinol, protease inhibitors, nitroglycerin) have yielded disappointing results, ${ }^{1,3-6}$ a few recent studies have shown significant benefit in reduction of PEP with administration of nonsteroidal anti-inflammatory drugs (NSAIDs). ${ }^{1,7,8}$ Recent meta-analyses have shown that prophylactic use of NSAIDs reduces the incidence and severity of PEP. ${ }^{9,10}$ Because the patient- and procedure-related factors affecting the efficacy of NSAIDs and the optimal timing, route of administration, and type of NSAID for PEP prophylaxis remain unclear, we have systematically evaluated and summarized available data on this topic.

\section{Materials and Methods}

\section{Search Strategy}

Two authors (T.R. and B.N.) independently conducted a comprehensive search of the Cochrane library, PubMed, and Scopus from January 1980 to November 2013. Clinical trial databases (www.clinicaltrials.gov) and published proceedings from major hepatology and gastrointestinal meetings in the past 5 years (including Digestive Disease Week, Canadian Digestive Disease Week, United European Gastroenterology Week, and American College of Gastroenterology and the Asia-Pacific Digestive Week) were also searched. Search terms included nonsteroidal anti-inflammatory drugs, diclofenac, indomethacin, aspirin, ibuprofen, naproxen, ketorolac, etodolac, sulindac, cyclooxygenase 2 inhibitors, celecoxib, rofecoxib, valdecoxib, pancreatitis, and ERCP. All relevant articles irrespective of language, year of publication, type of publication, or publication status were included. Data from quasirandomized or observational studies were excluded. The titles and abstracts of all potentially relevant studies were screened for eligibility. The reference lists of studies of interest were then manually reviewed for additional articles. In the case of studies with incomplete information, the principal authors were contacted to obtain additional data.

\section{Outcome Measures}

Our primary outcome measure was the overall incidence reduction of PEP in patients who received NSAIDs compared with that in patients who received placebo. In addition to overall outcome measures, subgroup analysis by type (indomethacin vs other NSAIDs), timing (before vs after ERCP), and route of administration (rectal vs other routes) was performed. We also performed additional exploratory subgroup analyses on the following patient and procedure characteristics: age, sex, sphincter of Oddi dysfunc tion (SOD), pancreatic sphincterotomy, and pancreatic duct injection. 


\section{Assessment of Risk of Bias in Included Studies}

The methodological quality of the trials, hence risk of bias, was assessed as follows: allocation sequence generation, allocation concealment, blinding, incomplete outcome data, selective outcome reporting, and vested interest bias. The instructions given in the Cochrane Handbook for Systematic Reviews of Interventions and the Cochrane Hepato-Biliary Group Module were followed. ${ }^{11,12}$

\section{Data Synthesis and Statistical Analysis}

Two independent reviewers extracted data and scored publications; a third investigator adjudicated discrepancies. The $\kappa$ scores were measured to assess the agreement between the 2 initial reviewers in each step and interpreted as described. ${ }^{13}$ We performed the review and meta-analyses following the recommendations of the Cochrane Collaboration. ${ }^{14}$ The analyses were performed using RevMan 5.2. The data were analyzed by intention-to-treat analysis, including all patients irrespective of compliance or follow-up. Binary outcomes were expressed as relative risks (RRs) with 95\% confidence intervals (CIs). Rare events were estimated by Peto odds ratio. Data were analyzed by fixed-effects or random-effects model depending on heterogeneity. ${ }^{15}$ Regression analyses were performed to estimate funnel plot asymmetry. ${ }^{16}$

In our analysis, heterogeneity was explored by the $\chi^{2}$ test, with significance set at a $P$ value of 0.10 and measured by $P^{2} \cdot{ }^{11}$ In the case of trials with zero events on clinical outcomes, we applied an empirical continuity correction of 0.5 in both arms to avoid overestimating a treatment effect. ${ }^{17}$ The preferred reporting items for systematic reviews and meta-analyses statement outline for reporting systematic reviews and meta-analyses was used to report this study. ${ }^{18}$

\section{Sensitivity Analysis}

A sensitivity analysis was conducted for every study to determine whether any single study was incurring undue weight in the analysis. We systematically removed 1 set of study data and checked the pooled results for the remaining studies to see if there was any significant change in test performance. Metaregression was also used to quantitatively explore possible reasons for heterogeneity in our subgroup analyses.

\section{Results}

\section{Characteristics of Included Studies}

Eighty-five potentially relevant studies were identified by our primary search of the electronic databases for published work on the subject and 6 additional records identified in a secondary search. Of these studies, 74 studies were screened after duplicate records were excluded. After further review of the title and abstract for irrelevant topics, an additional 63 records were excluded for meeting exclusion criteria $(n=59)$ or lack of randomization $(n=$ 4). The detailed process of this literature search is shown in Figure 1.

After careful review, 11 studies with 2497 patients (1254 in treatment group and 1243 in placebo group) were included in the meta-analysis. There was an excellent interreviewer 
agreement ( $\kappa=0.91 ; 95 \% \mathrm{CI}, 0.66-1.0)$. The characteristics of included studies are shown in Table 1. Figures 2A and B present the consensus risk of bias assessments of the included trials.

\section{Analysis of Outcomes}

There was an overall statistically significant decreased in the incidence of PEP for patients who received NSAIDs compared with that for patients who received placebo (RR, 0.59; $95 \%$ CI, $0.41-0.85 ; P=0.005 ;$ Fig. 3). The number needed to treat was 18 .

In our subgroup analysis by treatment type, 5 studies $(n=1539$; weight, $49.8 \%)$ using indomethacin showed no statistically significant difference (RR, 0.66 ; 95\% CI, $0.38-1.15 ; P$ $=0.14)$, but 6 studies $(\mathrm{n}=958$; weight, $50.2 \%)$ using other NSAIDS (all using diclofenac except 1 study using valdecoxib) showed significant benefit (RR, 0.51; 95\% CI, 0.29-0.91; $P=0.02$ ) in PEP prophylaxis (Fig. 3).

By administration route, only the rectal NSAIDs (7 studies with 1768 patients; weight, $62.4 \%$ ) showed significant overall reduction in incidence of PEP (RR, 0.47 ; 95\% CI, $0.35-$ $0.64 ; P<0.00001)$, whereas pooled data from 4 studies $(\mathrm{n}=651$; weight, $37.6 \%)$ with other routes of NSAID administration showed no significant benefit (RR, 1.04; 95\% CI, 0.57$1.87 ; P=0.91$ ) in reducing the risk of PEP (Fig. 4).

In subgroup analysis by timing of administration, pooled data from 4 studies $(\mathrm{n}=924$; weight, 37.4\%) in which NSAIDs were administered before ERCP showed statistically significant difference (RR, 0.48; 95\% CI, 0.29-0.78; $P=0.003$ ). However, 6 studies ( $=$ 1366; weight, 62.6\%) where NSAIDs were administered after procedure showed no significant benefit (RR, 0.62; 95\% CI, 0.34-1.11; $P=0.11$ ) in PEP prophylaxis (Fig. 5).

The results of our exploratory subgroup analyses are summarized in Table 2. Nonsteroidal anti-inflammatory drugs were effective in both young and old females and did not depend on whether a sphincterotomy was performed or not. Nonsteroidal anti-inflammatory drugs were also equally effective in patients who did not have a SOD/hypertension or pancreatic duct injection. On the contrary, NSAID use did not show a benefit in men and in patients who had a confirmed SOD/hypertension or pancreatic duct injection during the ERCP (Figs. 6A-E).

\section{Publication Bias}

Visual inspection of funnel plots showed that the studies were well scattered with no suggestion of any publication bias. The indicators for publication bias are the Begg adjusted rank correlation $(P=0.55)$ and Egger regression asymmetry tests $(P=0.43)$, which indicated no significant publication bias (Figs. 1A-C, Supplemental Digital Content, http:// links.lww.com/MPA/A357).

\section{Sensitivity Analysis}

Sensitivity analysis found that the study by Elmi et $\mathrm{al}^{21}$ was the source of the heterogeneity observed in our subgroup analyses. However, the prophylactic efficacy of NSAIDs was not affected after removing this study (RR, $0.55 ; 95 \% \mathrm{CI}, 0.40-0.75 ; P^{2}=27 \%, P=0.19$ ).

Similar results were obtained in our subgroup analyses by treatment type and administration 
route. However, after controlling for heterogeneity, in our subgroup analysis by timing of administration, both pooled data from 4 studies $(\mathrm{n}=924)$ in which NSAIDs were administered before ERCP (RR, 0.48; 95\% CI, 0.29-0.78; $P=0.003$ ) and 5 studies (n = 1249) where NSAIDs were administered immediately after the procedure showed significant benefit (RR, 0.52 ; 95\% CI, $0.33-0.80 ; P^{2}=10 \%, P=0.35$ ) in PEP prophylaxis.

Metaregression analysis did not show heterogeneity from study sample size $(\beta=-0.07 ; 95 \%$ CI, -0.31 to $0.17 ; P=0.33$ ) or publication year $(\beta=-0.02 ; 95 \% \mathrm{CI},-0.03$ to $0.21 ; P=$ $0.30)$.

\section{Discussion}

This meta-analysis of the 11 included randomized controlled trials show that patients undergoing ERCP who receive prophylactic NSAIDs are $41 \%$ less likely to have PEP. The number need to treat to prevent 1 episode of PEP is 18. Our findings are similar to previous meta-analyses published on this topic including the recent meta-analysis by Ding et al, ${ }^{9}$ which included 10 studies comprising 2269 patients. However, our meta-analysis is more current, and we performed subgroup analyses to evaluate the effect of type of NSAID, timing, and route of administration of NSAID on the prophylactic efficacy. We also performed exploratory subgroup analyses to identify patient- and procedure-related factors affecting the efficacy of NSAIDs in preventing PEP.

There were 3 kinds of NSAIDs used in these 11 trials-indomethacin, diclofenac, and valdecoxib. Although there are many different types of NSAIDs categorized according to their chemical structures, they share similar activities in inhibiting cyclooxygenase enzyme and reducing prostaglandin synthesis and have similar anti-inflammatory effects. However, we found significant difference in efficacy between indomethacin and other NSAIDs, predominantly diclofenac, in the prevention of PEP. Although the mechanisms of NSAIDs action seem to be similar, our analysis suggests that they differ in their ability to reduce PEP.

The findings of our study differ from those of recently published meta-analysis by Ding et al, ${ }^{9}$ which concluded that indomethacin and diclofenac had essentially the same effectiveness for PEP prophylaxis. Indeed, we found that pooled results from randomized controlled trials using indomethacin showed no significant benefit in preventing PEP but found a significant reduction in PEP in studies that used a nonindomethacin NSAID. This difference was observed despite the fact that indomethacin group had more patients (although similar weightage), a much higher proportion of patients who received the drug rectally ( 4 of 5 studies with 1422 of 1539 patients vs 3 of 6 studies with 424 of 958 patients; $P<0.00001)$, and a significantly higher number of patients who received NSAIDs before ERCP ( 3 of 5 studies with 820 of 1539 patients vs 1 of 6 studies with 104 of 958 patients; $P$ $<0.00001)$, compared with the nonindomethacin group. In addition, there was no significant difference in the proportion of patients with moderate to severe PEP in the 2 groups ( 3 of 5 studies with 46 of 1161 patients vs 3 of 6 studies with 18 of 531 patients; $P<0.68$ ), which could have accounted for this observed difference. Regarding cyclooxygenase 2 inhibitors, because only 1 study was available using valdecoxib (with negative results), a subgroup analysis of this NSAID type was not possible. ${ }^{20}$ 
Why differences in efficacy should exist between NSAIDS is unclear. Although both indomethacin and diclofenac belong to the arylalkanoic acids group of NSAIDs, small differences in their chemical structure could affect their efficacy, potency, and pharmacokinetic properties. Diclofenac has distinct features when compared with other NSAIDs. There is some evidence that it inhibits the lipoxygenase pathways, thus reducing formation of the leukotrienes, which are proinflammatory autacoids that have also been implicated as mediators of inflammation in acute pancreatitis. ${ }^{28-34} \mathrm{In}$ addition, it has been suggested that diclofenac is much more potent than other NSAID types in its ability to inhibit phospholipase A2, an enzyme strongly implicated in the pathogenesis of acute pancreatitis. These distinct effects of diclofenac may explain the significant efficacy of diclofenac in preventing PEP in this study.

The route of NSAID administration is also important factor affecting its clinical efficacy. From a clinical standpoint, all 7 studies assessing rectally administered NSAIDs to prevent PEP had positive results or demonstrated a trend toward positivity, whereas the results of 4 published studies assessing orally, intramuscularly, intravenously, and intraduodenally administered NSAIDs were negative. We compared the RR of rectal route of NSAID administration with nonrectal administration; only the rectal route showed significant benefit in preventing PEP. Although high first-pass metabolism of orally or intraduodenally administered drugs might explain the lack of efficacy, it is unlikely that this explains the negative results from parenterally (intramuscular or intravenous) administered drugs.

Regarding the optimal timing for administration, NSAID administration before the procedure (4 studies) was associated with a significant reduction in PEP. In 1 study, NSAIDs were administered both before and after the ERCP, and therefore it was excluded from this analysis. ${ }^{24}$ The time required for the onset of drug action, with lack of protection during the duration of procedure itself (intraprocedure) and immediately postprocedure period, is the likely explanation for the potentially greater benefit of preprocedure NSAIDS. Pancreatic acinar cell injury rapidly leads to a proinflammatory cascade with a very short potential therapeutic window for intervention. Once inciting injury, such as pancreatic duct cannulation and injection, initiates the inflammatory cascade, it might be difficult to halt its progression and prevent development of overt PEP.

The results of our exploratory subgroup analyses are interesting; however, they should be interpreted with caution because of the reduced power of some of the comparisons. Only few studies have compared the incidence of PEP in NSAID and control groups according to individual patient- and procedure-related risk factors. We found that both patients with and without pancreatic sphincterotomy showed prophylactic benefit from receiving NSAIDs, although patients without pancreatic sphincterotomy might be more likely to benefit. Although few studies and low number of patients may explain the nonsignificant results in male patients, lack of benefit in patients with SOD and pancreatic duct injection is likely real. Such patients are at high risk for developing PEP and might benefit from pancreatic stenting to reduce the incidence and severity of PEP.

Pancreatic duct stenting is another intervention that has been shown to reduce the incidence and severity of PEP. Papillary trauma and manipulation during ERCP can lead to sphincter 
of Oddi spasm and/or papillary edema, thus causing transient obstruction to the outflow of pancreatic juice. ${ }^{20}$ Randomized controlled trials have shown that prophylactic placement of a pancreatic duct stent in high-risk patients reduce the incidence of pancreatitis by mechanically facilitating pancreatic duct drainage ${ }^{20,35-37}$ However, pancreatic duct stenting is technically challenging, time-consuming, costly, and potentially dangerous if attempted but unsuccessful because it is associated with a high rate of PEP by causing pancreatic orifice injury but providing no ductal decompression. ${ }^{20,38-40}$ On the other hand, NSAIDs in addition to predominantly inhibiting the inflammatory cascade involved in the pathogenesis of acute pancreatitis also facilitate adequate drainage of the pancreatic duct by pharmacologically relieving papillary edema. ${ }^{20}$ Although mechanistically plausible that these interventions may complement one another by working in completely different ways, a recent network meta-analysis showed that combination of rectal NSAIDs and stents was not superior to either approach alone. ${ }^{41}$

Although our meta-analysis raises clinically relevant issues related to NSAIDs for preventing PEP, some limitations need to be acknowledged. The characteristics of included patients and intervention regimens varied among studies leading to some heterogeneity in our overall analyses. This feature of the patient cohorts could be a weakness and strength of the analysis. Although minimal variation of intervention regimen would have provided a more focused answer, the increased variation of patients and intervention regimens in our included studies increased the external validity of the results. Another possible limitation is that a proportion of the studies (4 of 11) were rated as low quality (Jadad scale score, 2). However, exclusion of low-quality studies did not change the risk reduction observed in any of the analyses. Lastly, additional randomized trials are necessary to directly compare different doses of indomethacin and diclofenac to determine the best NSAID and optimal dose of administration.

In conclusion, this meta-analysis shows that NSAIDs other than indomethacin are effective in preventing PEP. The rectal route compared with other routes of administration and administration before or immediately after ERCP seems to be most effective in the prevention of PEP. Based on these findings, diclofenac administered rectally before the ERCP could provide maximal prophylactic benefit for PEP, but it needs to be examined in prospective clinical studies.

\section{Supplementary Material}

Refer to Web version on PubMed Central for supplementary material.

\section{Acknowledgments}

The authors would like to thank Dr Fred S. Gorelick, Professor of Medicine, Section of Digestive Diseases, Yale University School of Medicine, New Haven, CT, for the critical appraisal of the study.

\section{References}

1. Rustagi T, Jamidar PA. Endoscopic retrograde cholangiopancreatography (ERCP)-related adverse events: post-ERCP pancreatitis. Gastrointest Endosc Clin N Am. 2015; 25:107-121. [PubMed: 25442962] 
2. Rustagi T, Jamidar PA. Endoscopic retrograde cholangiopancreatography-related adverse events: general overview. Gastrointest Endosc Clin N Am. 2015; 25:97-106. [PubMed: 25442961]

3. Sternlieb JM, Aronchick CA, Retig JN, et al. A multicenter, randomized, controlled trial to evaluate the effect of prophylactic octreotide on ERCP-induced pancreatitis. Am J Gastroenterol. 1992; 87:1561-1566. [PubMed: 1279967]

4. Andriulli A, Leandro G, Federici T, et al. Prophylactic administration of somatostatin or gabexate does not prevent pancreatitis after ERCP: an updated meta-analysis. Gastrointest Endosc. 2007; 65:624-632. [PubMed: 17383459]

5. Sherman S, Blaut U, Watkins JL, et al. Does prophylactic administration of corticosteroid reduce the risk and severity of post-ERCP pancreatitis: a randomized, prospective, multicenter study. Gastrointest Endosc. 2003; 58:23-29. [PubMed: 12838216]

6. Shao LM, Chen QY, Chen MY, et al. Nitroglycerin in the prevention of post-ERCP pancreatitis: a meta-analysis. Dig Dis Sci. 2010; 55:1-7. [PubMed: 19160042]

7. Elmunzer BJ, Scheiman JM, Lehman GA, et al. A randomized trial of rectal indomethacin to prevent post-ERCP pancreatitis. N Engl J Med. 2012; 366:1414-1422. [PubMed: 22494121]

8. Otsuka T, Kawazoe S, Nakashita S, et al. Low-dose rectal diclofenac for prevention of postendoscopic retrograde cholangiopancreatography pancreatitis: a randomized controlled trial. J Gastroenterol. 2012; 47:912-917. [PubMed: 22350703]

9. Ding X, Chen M, Huang S, et al. Nonsteroidal anti-inflammatory drugs for prevention of post-ERCP pancreatitis: a meta-analysis. Gastrointest Endosc. 2012; 76:1152-1159. [PubMed: 23164513]

10. Ahmad D, Lopez KT, Esmadi MA, et al. The effect of indomethacin in the prevention of postendoscopic retrograde cholangiopancreatography pancreatitis: a meta-analysis. Pancreas. 2014; 43:338-342. [PubMed: 24622061]

11. Zheng MH, Xia HH, Chen YP. Rectal administration of NSAIDs in the prevention of post-ERCP pancreatitis: a complementary meta-analysis. Gut. 2008; 57:1632-1633. [PubMed: 18941015]

12. Klingenberg SL, Nikolova D, Alexakis N, et al. Hepato-biliary clinical trials and their inclusion in the Cochrane Hepato-Biliary Group register and reviews. J Gastroenterol Hepatol. 2011; 26:649656. [PubMed: 21418299]

13. Viera AJ, Garrett JM. Understanding interobserver agreement: the kappa statistic. Fam Med. 2005; 37:360-363. [PubMed: 15883903]

14. Cheon YK. Can postendoscopic retrograde cholangiopancreatography pancreatitis be prevented by a pharmacological approach? Korean J Intern Med. 2013; 28:141-148. [PubMed: 23525264]

15. DerSimonian R, Laird N. Meta-analysis in clinical trials. Control Clin Trials. 1986; 7:177-188. [PubMed: 3802833]

16. Egger M, Davey Smith G, Schneider M, et al. Bias in meta-analysis detected by a simple, graphical test. BMJ. 1997; 315(7109):629-634. [PubMed: 9310563]

17. Sweeting MJ, Sutton AJ, Lambert PC. What to add to nothing? Use and avoidance of continuity corrections in meta-analysis of sparse data. Stat Med. 2004; 23:1351-1375. [PubMed: 15116347]

18. Liberati A, Altman DG, Tetzlaff J, et al. The PRISMA statement for reporting systematic reviews and meta-analyses of studies that evaluate health care interventions: explanation and elaboration. Ann Intern Med. 2009; 151:W65-W94. [PubMed: 19622512]

19. Döbrönte Z, Toldy E, Márk L, et al. Effects of rectal indomethacin in the prevention of post-ERCP acute pancreatitis [in Hungarian]. Orv Hetil. 2012; 153:990-996. [PubMed: 22714033]

20. Bhatia V, Ahuja V, Acharya SK, et al. A randomized controlled trial of valdecoxib and glyceryl trinitrate for the prevention of post-ERCP pancreatitis. J Clin Gastroenterol. 2011; 45:170-176. [PubMed: 20717044]

21. Elmi F, Rossi F, Lim JK, et al. A prospective, multicenter, randomized, double blinded controlled study to determine whether a single dose of intraduodenal indomethacin can decrease the incidence and severity of post-ERCP pancreatitis. Gastrointest Endosc. 2010; 71:AB232.

22. Senol A, Saritas U, Demirkan H. Efficacy of intramuscular diclofenac and fluid replacement in prevention of post-ERCP pancreatitis. World J Gastroenterol. 2009; 15:3999-4004. [PubMed: 19705494] 
23. Khoshbaten M, Khorram H, Madad L, et al. Role of diclofenac in reducing post-endoscopic retrograde cholangiopancreatography pancreatitis. J Gastroenterol Hepatol. 2008; 23(7 Pt 2):e11e16. [PubMed: 17683501]

24. Cheon YK, Cho KB, Watkins JL, et al. Efficacy of diclofenac in the prevention of post-ERCP pancreatitis in predominantly high-risk patients: a randomized double-blind prospective trial. Gastrointest Endosc. 2007; 66:1126-1132. [PubMed: 18061712]

25. Sotoudehmanesh R, Khatibian M, Kolahdoozan S, et al. Indomethacin may reduce the incidence and severity of acute pancreatitis after ERCP. Am J Gastroenterol. 2007; 102:978-983. [PubMed: 17355281]

26. Montaño Loza A, Rodríguez Lomelí X, García Correa JE, et al. Effect of the administration of rectal indomethacin on amylase serum levels after endoscopic retrograde cholangiopancreatography, and its impact on the development of secondary pancreatitis episodes [in Spanish]. Rev Esp Enferm Dig. 2007; 99:330-336. [PubMed: 17883296]

27. Murray B, Carter R, Imrie C, et al. Diclofenac reduces the incidence of acute pancreatitis after endoscopic retrograde cholangiopancreatography. Gastroenterology. 2003; 124:1786-1791. [PubMed: 12806612]

28. Folch E, Closa D, Prats N, et al. Leukotriene generation and neutrophil infiltration after experimental acute pancreatitis. Inflammation. 1998; 22:83-93. [PubMed: 9484652]

29. Cartmell MT, O'Reilly DA, Porter C, et al. A double-blind placebo-controlled trial of a leukotriene receptor antagonist in chronic pancreatitis in humans. J Hepatobiliary Pancreat Surg. 2004; 11:255-259. [PubMed: 15368110]

30. Hirano T. Peptide leukotriene receptor antagonist diminishes pancreatic edema formation in rats with cerulein-induced acute pancreatitis. Scand J Gastroenterol. 1997; 32:84-88. [PubMed: 9018772]

31. Oruc N, Yukselen V, Ozutemiz AO, et al. Leukotriene receptor antagonism in experimental acute pancreatitis in rats. Eur J Gastroenterol Hepatol. 2004; 16:383-388. [PubMed: 15028970]

32. Ozkan E, Akyuz C, Sehirli AO, et al. Montelukast, a selective cysteinyl leukotriene receptor 1 antagonist, reduces cerulein-induced pancreatic injury in rats. Pancreas. 2010; 39:1041-1046. [PubMed: 20467345]

33. Vigna SR, Shahid RA, Nathan JD, et al. Leukotriene B4 mediates inflammation via TRPV1 in duct obstruction-induced pancreatitis in rats. Pancreas. 2011; 40:708-714. [PubMed: 21602738]

34. Vollmar B, Waldner H, Schmand J, et al. Release of arachidonic acid metabolites during acute pancreatitis in pigs. Scand J Gastroenterol. 1989; 24:1253-1264. [PubMed: 2602907]

35. Fazel A, Quadri A, Catalano MF, et al. Does a pancreatic duct stent prevent post-ERCP pancreatitis? A prospective randomized study. Gastrointest Endosc. 2003; 57:291-294. [PubMed: 12612504]

36. Singh P, Das A, Isenberg G, et al. Does prophylactic pancreatic stent placement reduce the risk of post-ERCP acute pancreatitis? A meta-analysis of controlled trials. Gastrointest Endosc. 2004; 60:544-550. [PubMed: 15472676]

37. Tarnasky PR. Mechanical prevention of post-ERCP pancreatitis by pancreatic stents: results, techniques, and indications. JOP. 2003; 4:58-67. [PubMed: 12555017]

38. Freeman ML. Prevention of post-ERCP pancreatitis: pharmacologic solution or patient selection and pancreatic stents? Gastroenterology. 2003; 124:1977-1980. [PubMed: 12806633]

39. Freeman ML, Overby C, Qi D. Pancreatic stent insertion: consequences of failure and results of a modified technique to maximize success. Gastrointest Endosc. 2004; 59:8-14. [PubMed: 14722540]

40. Elmunzer BJ, Waljee AK. Can rectal NSAIDs replace prophylactic pancreatic stent placement for the prevention of post-ERCP pancreatitis? Gastroenterology. 2014; 146:313-315. discussion 315. [PubMed: 24269561]

41. Akbar A, Abu Dayyeh BK, Baron TH, et al. Rectal nonsteroidal anti-inflammatory drugs are superior to pancreatic duct stents in preventing pancreatitis after endoscopic retrograde cholangiopancreatography: a network meta-analysis. Clin Gastroenterol Hepatol. 2013; 11:778783. [PubMed: 23376320] 


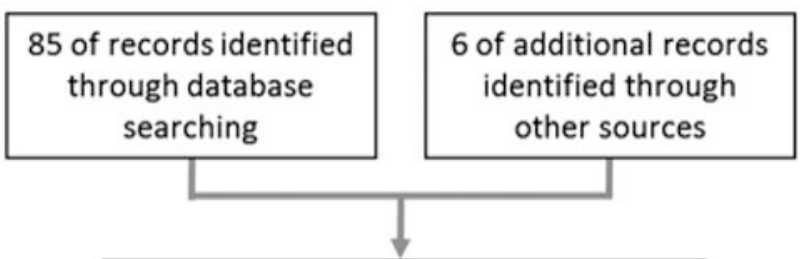

74 of records after duplicates removed

74 of records screened

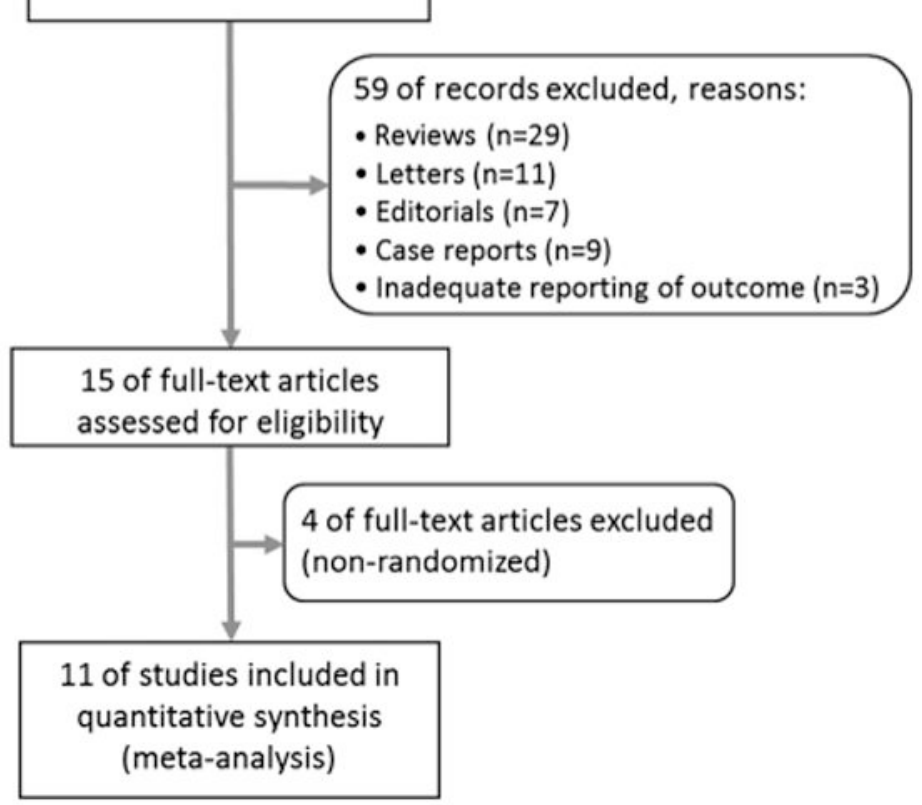

Figure 1.

Preferred reporting items for systematic reviews and meta-analyses flow chart describing the literature search conducted for this meta-analysis. 

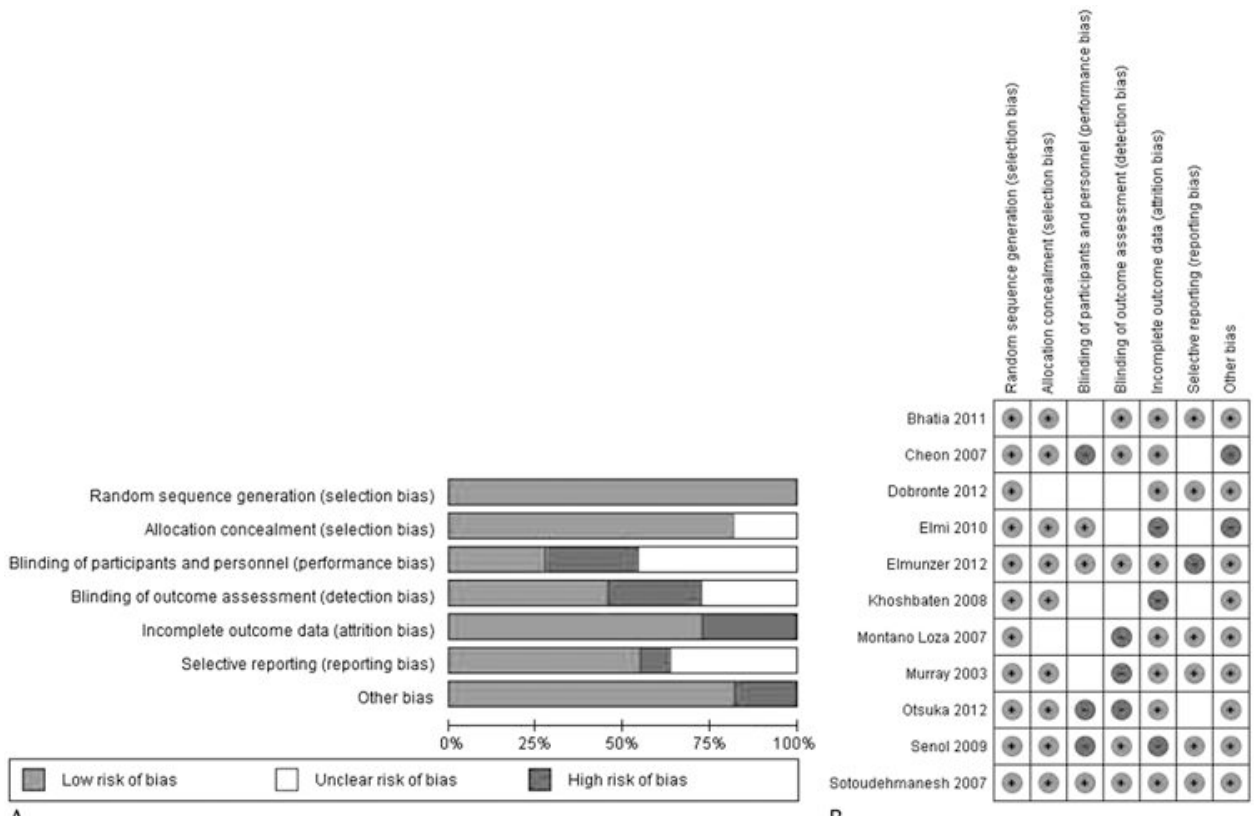

Figure 2.

Risk of bias graph review authors' judgements about each risk of bias item presented as percentages across all included studies (A). Risk of bias summary/consensus risk of bias assessments (B). 


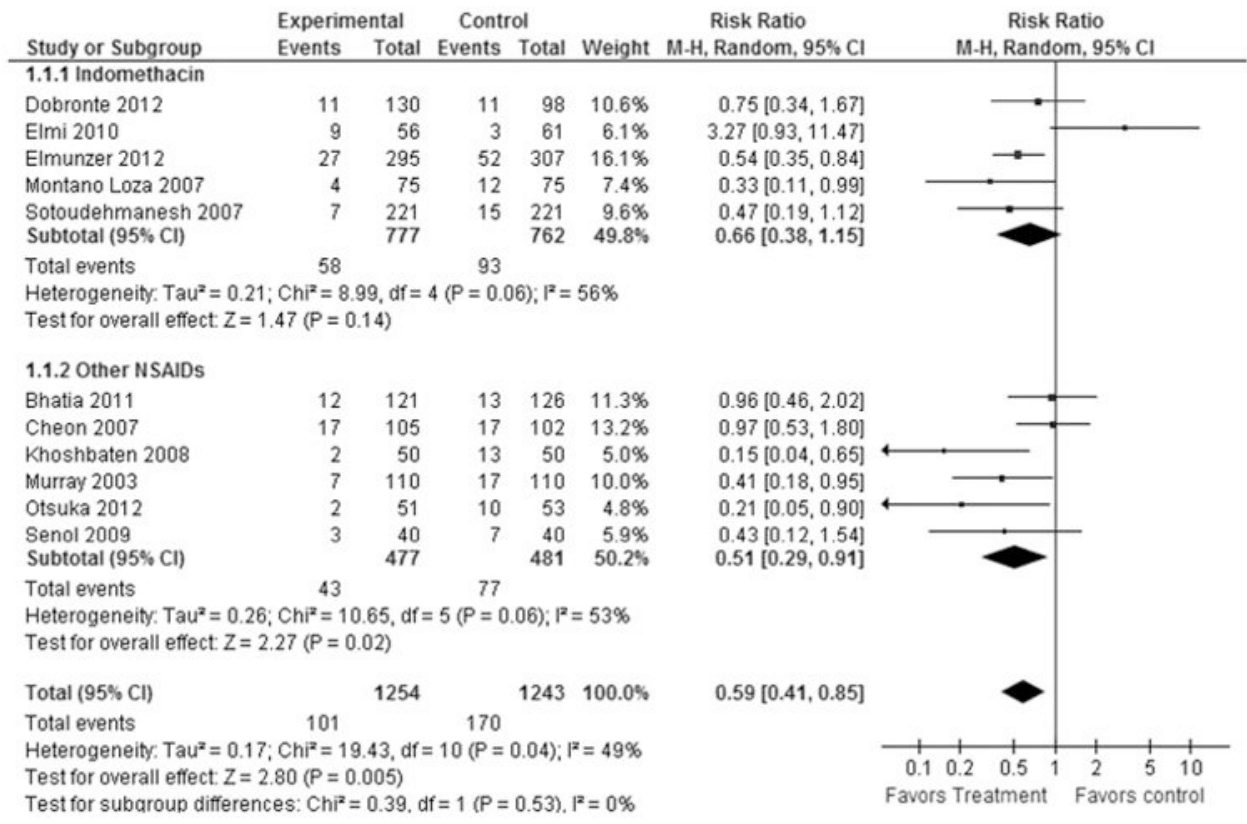

Figure 3.

Forest plot showing a significant difference in PEP prophylaxis by type of NSAID used. 


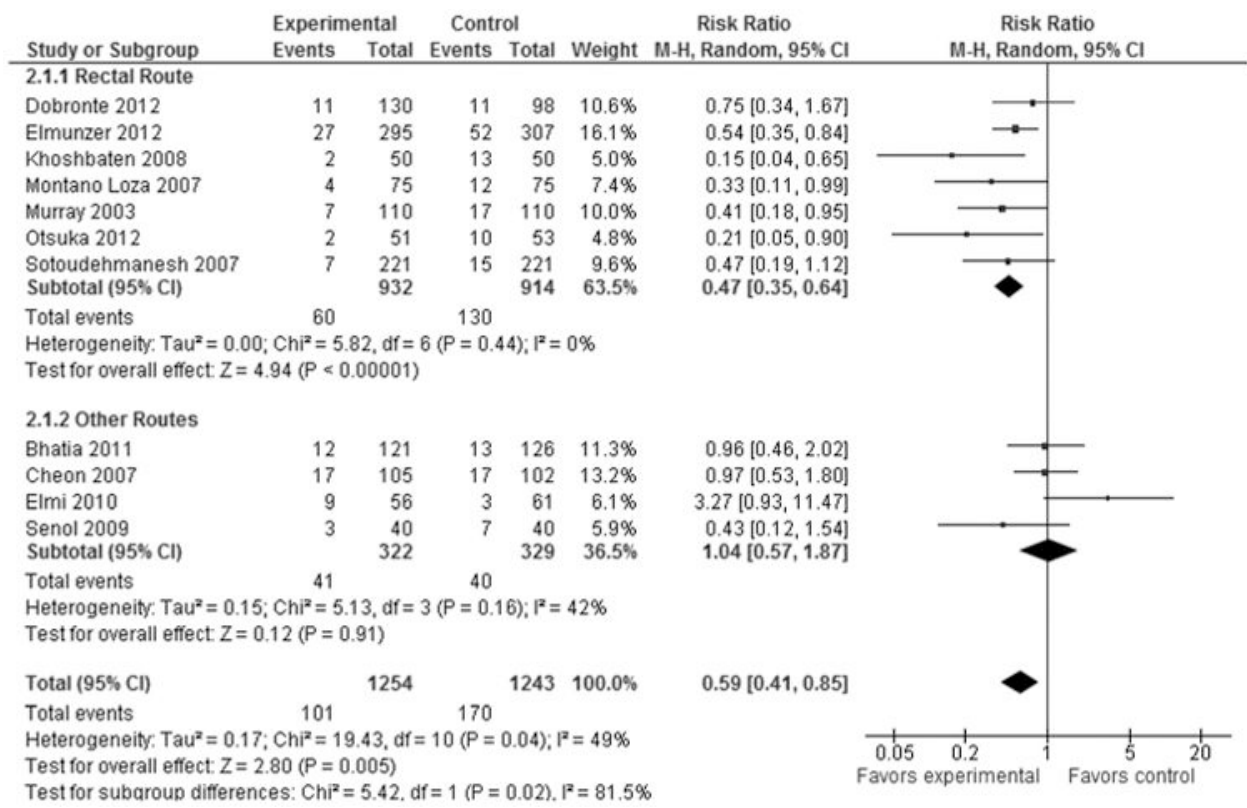

Figure 4.

Forest plot demonstrating a significant difference in PEP prevention by route of NSAID administration. 


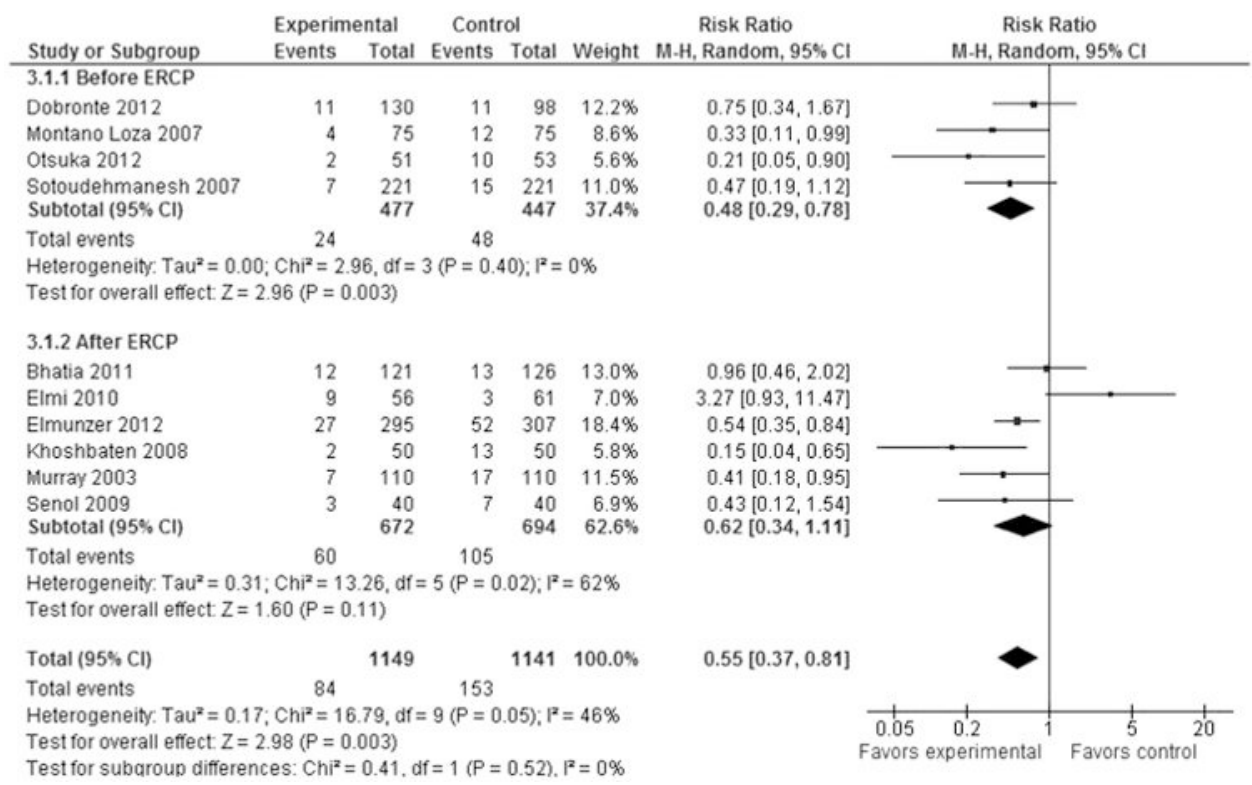

Figure 5.

Forest plot showing a significant difference in PEP prophylaxis by timing of NSAID administration. 

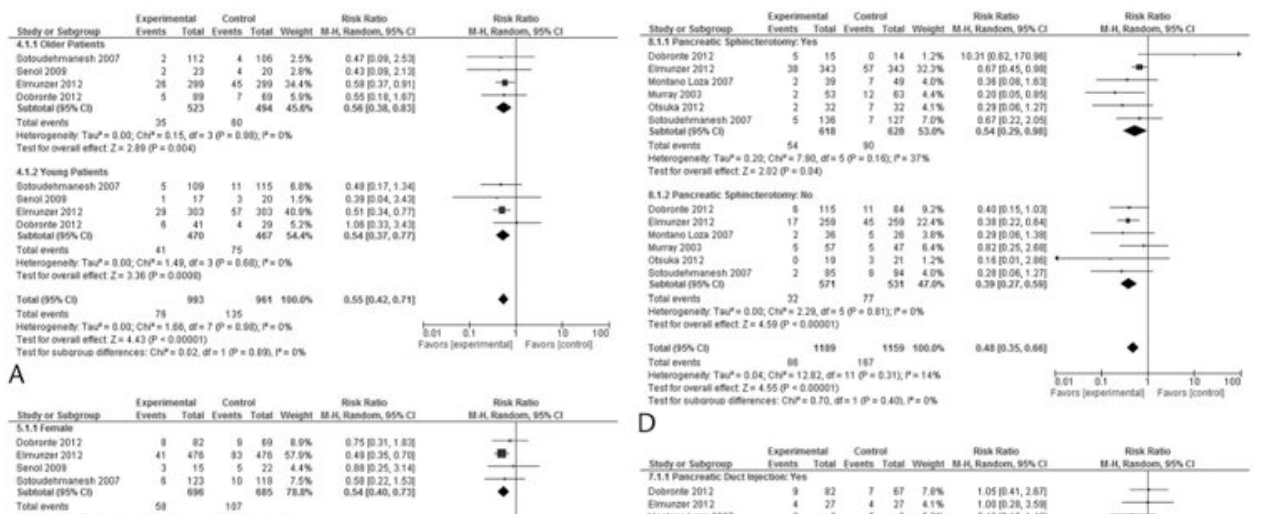

D

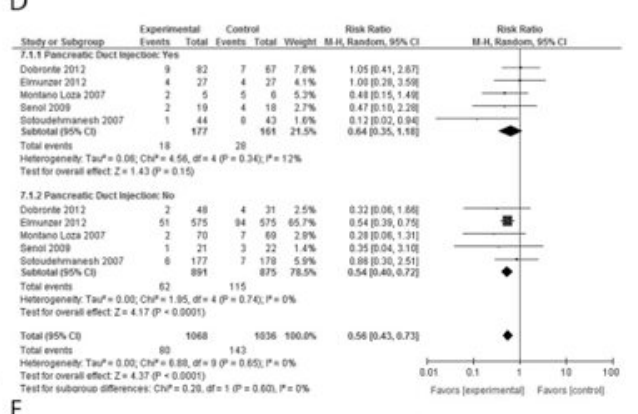

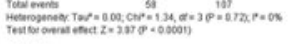

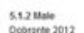

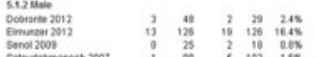

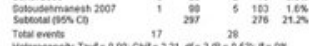

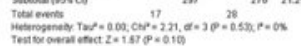

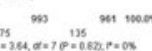

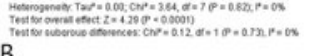

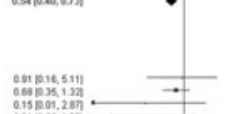

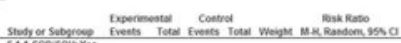

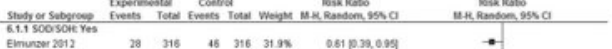

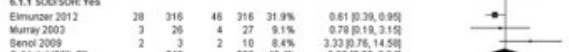

$\mathrm{E}$

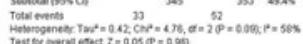

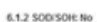

6.2 soosone
Elmurees 2012

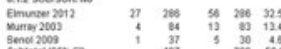

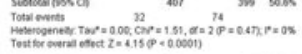

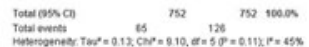

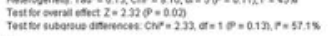

C

o.

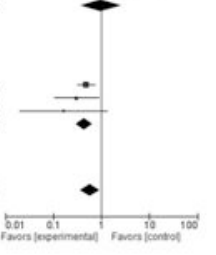

Figure 6.

Forest plot comparing efficacy of NSAIDs in preventing PEP based on age (A), sex (B), SOD (C), pancreatic sphincterotomy (D), and pancreatic duct injection (E). 


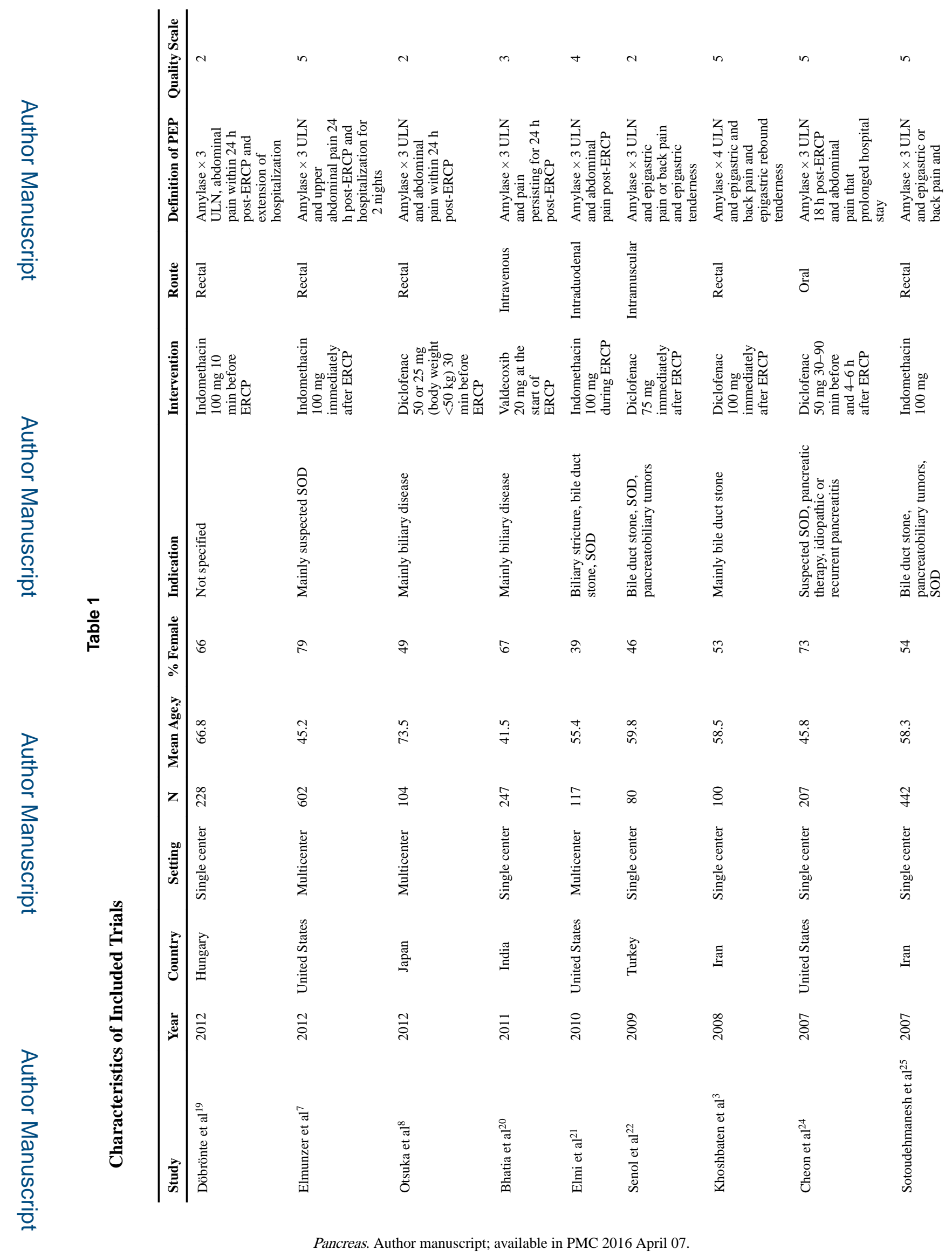


Rustagi and Njei

Page 17

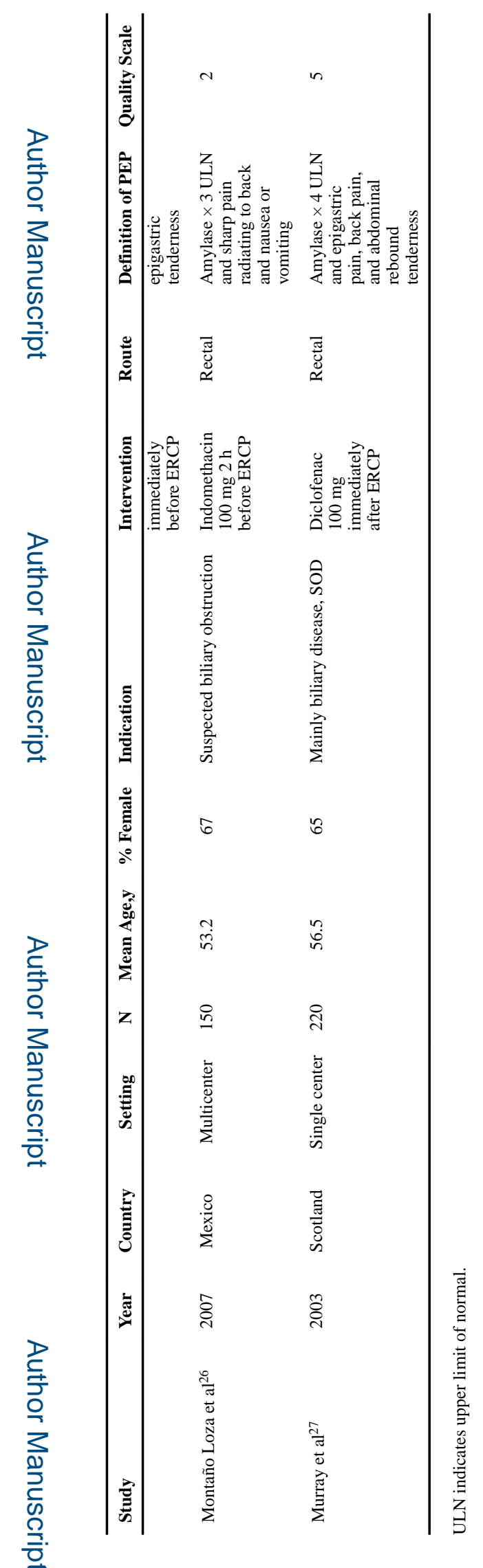

Pancreas. Author manuscript; available in PMC 2016 April 07. 
Table 2

Exploratory Subgroup Analyses: NSAIDs Versus Placebo in Reducing the Number of Pancreatitis

\begin{tabular}{lccc}
\hline Subgroup & No. Studies & No. Patients & RR $(\mathbf{9 5 \%} \mathbf{C I})$ \\
\hline Age & & & \\
$\quad$ Young ${ }^{*}$ & 4 & 937 & $0.54(0.37-0.77)$ \\
$\quad$ Old & & 1017 & $0.56(0.38-0.83)$ \\
Sex & 4 & 1381 & $0.54(0.40-0.73)$ \\
$\quad$ Female & 4 & 573 & $0.61(0.34-1.09)$ \\
$\quad$ Male & & & \\
SOD & 3 & 698 & $0.98(0.38-2.54)$ \\
$\quad$ Yes & 3 & 806 & $0.44(0.30-0.65)$ \\
$\quad$ No & & & \\
Pancreatic sphincterotomy & 6 & 1246 & $0.54(0.29-0.98)$ \\
$\quad$ Yes & 6 & 1102 & $0.39(0.27-0.59)$ \\
$\quad$ No & & & \\
Pancreatic duct injection & 5 & 338 & $0.64(0.35-1.18)$ \\
$\quad$ Yes & 5 & 1766 & $0.54(0.40-0.72)$ \\
$\quad$ No & &
\end{tabular}

